\title{
Preface to the special issue on advanced materials for nuclear energy applications
}

\author{
Guang-Hong $\mathrm{Lu}^{1} \cdot$ Fei Gao ${ }^{2}$ \\ (C) The Nonferrous Metals Society of China 2021 / Published online: 29 March 2021
}

Nuclear energy is playing an essential role in modern human society. Because of renewed public concern with regard to energy generation and climate change, worldwide interest in nuclear energy and technology is increasing. According to Director General Rafael Mariano Grossi from International Atomic Energy Agency (IAEA), nuclear energy contributes to $\sim 10 \%$ global energy. The development of advanced nuclear energy systems, including both fusion and fission, is significantly accelerated. The largest fusion device under construction, ITER, is now preparing to install the first coil pair. The Joint European Torus (JET) has recently started fusion experiments using tritium, a radioactive isotope of hydrogen which is very rare and expensive. In China, Hualong One, the Chinese Generation III + pressurized water nuclear reactor, enters commercial service at the beginning of 2021. As the power capability keeps increasing in these advanced nuclear energy systems, the materials in these nuclear systems are exposed to aggressive environmental conditions and damage from high-temperature radiation, which results in mechanical degradation and life reduction. As a highly active field, both challenges and opportunities are present, and thus, we encourage the youth and talents to join the field, contributing to the development of advanced nuclear materials.

This special topic issue is about advanced materials for nuclear energy applications, especially considering the neutron radiation effect. It includes contributions from researchers from various countries including China, Canada, Germany, Japan and United States, consisting of

Guang-Hong Lu

LGH@buaa.edu.cn

Fei Gao

gaofeium@umich.edu

1 School of Physics, Beihang University, Beijing 100191, China

2 Department of Nuclear Engineering and Radiological Sciences, University of Michigan, Ann Arbor, MI 48109, USA three review papers and five research articles. Three contributions are about the development of advanced tungstenbased materials including nanostructured tungsten, self-passivating smart tungsten alloys and selective laser melting ZrC-reinforced tungsten. Prof. Xiang-Heng Xiao reviews the development status of nanostructured tungsten and the performance enhancement mechanism of diverse nanostructure. Prof. Andrey Litnovsky, a senior scientist from Forschungszentrum Jülich reports the progress in joining and upscale of self-passivating smart tungsten alloys for DEMO first wall mockups. Dr. Yan Zhou from China University of Geosciences presents the selective laser melting high-performance ZrC-reinforced tungsten composites, which suppresses the cracking susceptibility of elective laser melting in pure tungsten. The neutron radiation effect is discussed in both experimental and computational efforts. Prof. Toshimasa Yoshiie from Kyoto University reviews the experimental results on void swelling in austenitic stainless steels and highlights the role of alloying elements. Prof. Zhong-Wen Yao from Queen's University discovered the delayed irradiation-induced phase instability in a $\mathrm{NiCr}$ FeTiNbAl alloy due to helium by means of in situ transmission electron microscopy (TEM). Dr. Xing-Zhong Cao from Institute of High Energy Physics, Chinese Academy of Sciences, presents thermal evolution of defects in tungsten and tungsten-rhenium alloy using the positron annihilation spectroscopy technic. Dr. Yuri N. Osetsky, a senior scientist from Oak Ridge National Laboratory, reports the results of an extensive atomic-scale modeling study of the interactions between moving edge dislocations and voids in tungsten, and Dr. Peng-Bo Zhang from Dalian Maritime University reviews the first-principles modeling of solute-point defect interactions in vanadium and its alloy.

We hope this issue could provide a clear insight about the development of advanced tungsten-based materials and recent research progress regarding neutron radiation effects and triggers potential interest for future research. At last, we want to thank all the authors, reviewers, editors, and 
publishing staff for their efforts to achieve the publication of this issue.

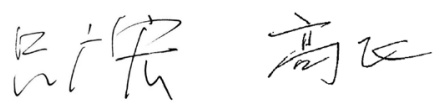

Dr. Guang-Hong Lu

Dr. Fei Gao

March 10, 2021

Publisher's Note Springer Nature remains neutral with regard to jurisdictional claims in published maps and institutional affiliations.

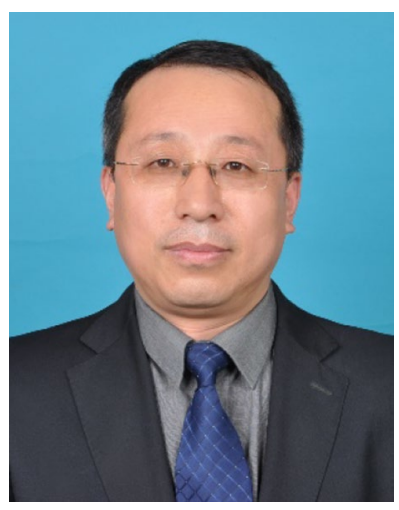

Dr. Guang-Hong Lu got his bachelor and master's degree from Jilin University, China, and $\mathrm{Ph} . \mathrm{D}$. from University of Tokyo, Japan, followed by working as a JSPS Foreign researcher in University of Tokyo and a research assistant professor in University of Utah, USA. Currently, he works as a professor in School of Physics, Beihang University, China. His main research focuses on metallic materials for applications of future nuclear fusion reactor.

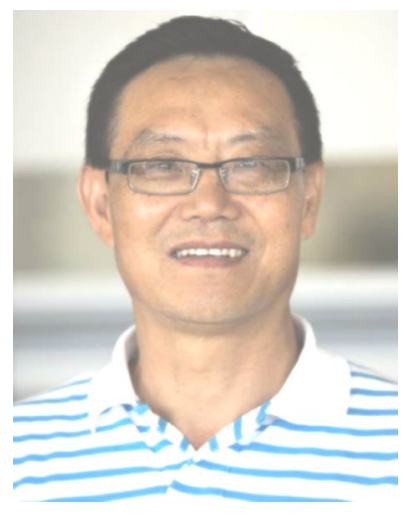

Dr. Fei Gao received his bachelor and master degrees from Lanzhou University, and his Ph.D. degree in Material Science and Engineering from the University of Liverpool of UK. Since then, he worked at University of Liverpool and Pacific Northwest National Laboratory of USA (PNNL) as senior research scientist and chief scientist, as well as an adjunct professor of Washington State University of USA. Since 2014, Dr. Gao joined the Department of Nuclear Engineering and Radiological Sciences in University of Michigan. Dr. Gao is now a full professor in both Department of Nuclear Engineering and Radiological Sciences and Department of Material Science and Engineering, University of Michigan, USA. His current work focuses on ion-solid interaction, irradiation damage, detector materials, nanostructure properties, Li-ion battery and multi-scale modeling of materials. 